
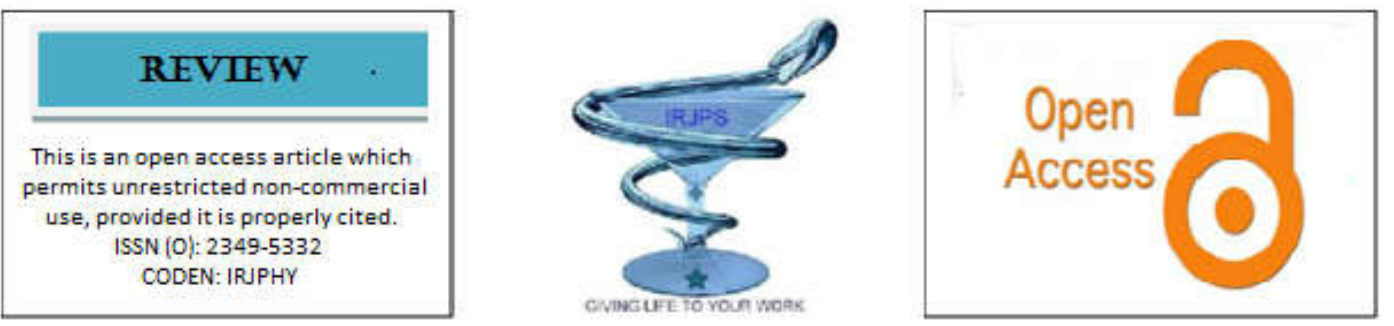

\title{
Pathogenesis of COVID-19
}

\section{Saumendu Deb Roy}

Department of Pharmaceutical Science, Mata Gujri University, Kishanganj, Bihar, India

\begin{abstract}
Pathogenesis of a disease is the biological mechanism involved in development of the disease. COVID-19 is a typical viral pneumonia with severe acute respiratory syndrome and hence the causative organism is called (SARS-CoV-2). The disease is highly fatal due to its respiratory complications and thus poses a global threat. Since the beginning of 2020 COVID-19 has caused a huge number of mortality and the rate is increasing ever since, thus the scientific community is engaged in lookout for prevention and possible treatment of the disease. For this, better understanding of pathogenesis of the disease is a prerequisite and this manuscript is a step towards understanding the pathogenesis of the disease.
\end{abstract}

KEY WORDS: Coronavirus, COVID-19, SARS-CoV-2, Pathogenesis, Virus

Corresponding Author: S Deb Roy

E-mail: baharu75@gmail.com

Mobile: +91- 7002107995
Indian Research Journal of Pharmacy and Science; 26(2020)2354-2358; Journal Home Page: https://www.irjps.in DOI: $10.21276 /$ irjps.2020.7.3.3 


\section{INTRODUCTION:}

Coronavirus (SARS-CoV-2) is considered to be one of the most deadly pathogen of recent past targeting the human respiratory system. From earlier outbreaks Severe acute respiratory syndromecoronavirus (SARS-CoV) and Middle East respiratory syndrome-coronavirus (MERS$\mathrm{CoV}$ ) were characterised as threat to public health [1,2]. In late 2019, SARS-CoV-2 registered its emergence in Wuhan, Hubei Province, of China and it rapidly spread to other areas, it was then discovered that a new variety of coronavirus was responsible and was named Novel (New) Coronavirus or COVID-19 by WHO on Feb 11, 2020 [3]. High virulence, lack of vaccines and specific antiviral led to spread of the disease worldwide whereas the large asymptomatic population turned out to be super spreaders, giving it the shape of Pandemic.

Coronavirus (SARS-CoV-2) is also an enveloped virus like (SARS-CoV) and (MERS-CoV) with single stranded RNA genome [4]. Virus genome sequencing of five patients from Wuhan, China suffering from the pneumonia in December 2019, revealed the causative organism to be a new unknown $\beta$ $\mathrm{CoV}$ strain, which has shown $88 \%$ similarity with the two bat-SARS-CoV, bat-SLCoVZC45 and bat-SL-CoVZXC21 and around $50 \%$ similarity with MERS-CoV. The genome of SARS-CoV-2 is somewhat similar to that of coronavirus. It also contains a minimum of 10 open reading frames [5].

Many research groups from China reported that SARS-CoV-2 also require Angiotensin Converting Enzyme 2 (ACE2) to enter cells and binding of virus with ACE2 is significant for pathogenesis of infection [6].

\section{SYMPTOMS:}

Symptoms of SARS-CoV-2 or COVID-19, like all other infectious diseases appears after an incubation period. The incubation period for COVID-19 is approximately 5.2 days [7].
Depending on age of patients and the status of his immune system, the days from infection to occurrence of symptoms and to death range from 0-6-41 days [8].

Most common symptoms of SARSCoV-2 include fever, cough and fatigue, which start on the onset of the disease. Others include aches and pains, sore throat, diarrhoea, conjunctivitis, headache, loss of taste and smell, skin rashes and discolouration of fingers and toes. Again the serious symptom includes difficulty in breathing, chest pain and loss of speech or movement [9].

SARS-CoV-2 shows symptoms similar to that of SARS-CoV or MERS-CoV, like fever, dry cough \& dyspnea, but it also has shown some new clinical features like targeting the lower airway which is justified by symptoms like rhinorrhoea, sneezing \& sore throat $[10,11]$.

The symptoms may in short be grouped in two classes-[9]

A) Systemic Symptoms

-Fever, Cough, Fatigue, Headache, Haemoptysis, Acute Cardiac injury, Hypoxemia, Dyspnoea, Lymphopenia \& Diarrhoea.

B) Respiratory Symptoms

-Rhinorrhoea, Sneezing, Sore Throat, Pneumonia, Ground-glam opacities, RN Aaemia \& Accute respiratory distress syndrome (ARDS)

\section{PATHOGENESIS:-}

Coronavirus responsible for COVID 19 is an enveloped positive sense, single stranded RNA virus of $26-32 \mathrm{~kb}$, and it also along with other $\mathrm{CoV}$ strains are capable of infecting a wide variety of species [4]. $\alpha \& \beta$ genera of $\mathrm{CoV}$ can infect humans, $\alpha \mathrm{CoV}$ like 229E \& NL63 can cause common cold and lyrangotracheo bronchitis. SARS-CoV, MERS-CoV and SASR $-\mathrm{CoV}-2$ are $\beta-\mathrm{CoV}$ [12]

SARS-CoV-2 within the host cell goes through five different phases; attachment, 
penetration, biosynthesis, maturation \& release. The virus binds with the receptor (ACE2) \& then enters the host cell through membrane fusion or endocytosis. The Virus then releases its contents in the host cell \& the RNA of virus enters the host cell nucleus for replication. Here the viral m-RNA prepares the viral protein $\&$ then new viral matter matures and is released. [12]

In host cell the activity of virus can be again subdivided into 3 phases depending upon the various clinical manifestations:

$1^{\text {st }}$ Initial Phase (1-2 days): In this stage the virus bind, to nasal epithelial cells \& replicates, and the virus spike glycoprotein binds to ACE2. Then the virus propagates and at this stage it can be found in nasal swabs. Here the host will be asymptomatic due to low viral burden, but can infect others $[13,14]$.

\section{$2^{\text {nd }}$ Acute or Intermediate phase (3-8} days): In this stage the Virus propagates \& than moves along the respiratory tract through conducting airways triggering robust immune response. Here the antigen presentation stimulates humoral \& cellular immunity by specific B \& $\mathrm{T}$ cells. Against SARS-CoV-2 $\mathrm{IgM}$ are $\mathrm{IgG}$ are produced, and among these IgM lasts for 12 week but $\mathrm{IgG}$ lasts long and plays protective role $[14,15]$. Nasal Swab \& Throat Swab at this stage shows the presence of the virus.

The interferon responsive gene CXCL 10 gene is an established marker in Covid 19, with a good signal to noise ration in alveolar II cell response in SARS-Cov-2.

$3^{\text {rd }}$ Severe Stage: Around $15-22 \%$ of the patients reaches this stage with symptoms like Hypoxia, Ground-glass opacification, which may lead to Acute respiratory distress syndrome (ARDS). SARS-Cov-2 in this stage reaches lungs \& infects alveolar cells, where type II cells are more prone to infection than type I cells, [16, 17]. SARS- CoV-2 here multiplies within type II cells, releases viral matters in large volume \& this leads to Apoptosis, [18].

Scientific findings suggest ARDS to be one of the main causes of Covid-19 death [19]. One of the major causative factor for ARDS is found to be cytokine storm \& uncontrolled systemic inflammatory response due to release of cytokines and chemokines [19]. This uncontrolled release triggers severe immune response causing ARDS, multiple organs failure \& finally death, in almost $2 \%$ of patients [18]. High proportion of aberrant coagulation is also noticed in critical patients, which may lead to overt disseminated intravascular coagulation (DIC) [19].

Corona viruses in general uses various strategies to avoid host immune responses. SARS-CoV under goes replication escaping the anti-viral immunity, [17]. The open reading frame 8 (ORF 8) protein disrupts antigen presentation of SARS-CoV infected cells, [18]. This suggests that ceasing the functioning of ORF 8 could be a strategy in the fight against Covid-19.

\section{DISCUSSION:}

The complications of Covid-19 arise due to interaction between SARS-CoV-2 \& host immune system. Viral factors \& Host immune system factors are responsible and all contribute to infection, severity of disease etc.

Studies so far reported that people aged 65 and above are more prone to infection, which in some reports were related to diminished immunity. With a mean incubation period of 5.2 days \& fatality rate of 2.3 the Pathogenesis needs to be studied more in molecular level to prevent the spread and rescue the ailing human race.

\section{REFERENCES:}

[1] A. Bogoch, A. Watts, Thomas-Bachli, C. Huber, M.U.G. Kraemer, K. Khan, Pneumonia 
of unknown etiology in wuhan, China: potential for international spread via commercial air travel, J. Trav. Med. (2020), https://doi.org/10.1093/jtm/taaa008.

[2] H. Lu, C.W. Stratton, Y.W. Tang, Outbreak of pneumonia of unknown etiology in wuhan China: the mystery and the miracle, J. Med. Virol. 92 (4) (2020) 401-402, https://doi.org/10.1002/jmv.25678.

[3] P. Zhou, X.L. Yang, X.G. Wang. A pneumonia outbreak associated with a new coronavirus of probable bat origin. Nature (2020), http://doi.org/10.1038/s4158G-0202012-7.

[4] S. Su, G. Wong, W. Shi, et.al., Epidemiology, Genetic recombination, and pathogenesis of coronavirus. Trends Microbiol. $24 \quad$ (2016) 490-502, https://doi.org/10.1016/j.tim.2016.03.003.

[5] X. Li, M. Geng, Y. Peng, L. Meng, S. Lu, Molecular immune pathogenesis and diagnosis of COVID- 19. J. Pharm. Ana. 10(2020) 102108 ,

https://doi.org/10.1016/j.jpha.2020.03.001.

[6] W. Li, M.J. Moore, N. Vasilieva, et.al., Angiotensin-converting enzyme 2 is a functional receptor for SARS coronavirus, Nature $\quad 426 \quad$ (2003) 450-454, https://doi.org/10.1038/nature02145.

[7] Q. Li, X. Guan, P. Wu, X. Wang, L. Zhou, $Y$. Tong, et al., Early transmission dynamics in wuhan, China, of novel coronavirus-infected pneumonia, N. Engl. J. Med. (2020), https://doi.org/10.1056/NEJMoa2001316.

[8] Q. Li, X. Guan, P. Wu, X. Wang, L. Zhou, Y. Tong, et al., Early transmission dynamics in wuhan, China, of novel coronavirus-infected pneumonia, N. Engl. J. Med. (2020), https://doi.org/10.1056/NEJMoa2001316.

[9] H.A. Rothana, S.N. Byraredd, The epidemiology and pathogenesis of coronavirus disease (COVID-19) outbreak, J. Autoimmun
109

(2020)

https://doi.org/10.1016/j.jaut.2020.102433.

[10] A. Assiri, J.A. Al-Tawfiq, A.A. AlRabeeah, F.A. Al-Rabiah, S. Al-Hajjar, A. Al Barrak, etal., Epidemiological,demographic, andclinicalcharacteristics of 47 cases of Middle East respiratory syndrome coronavirus disease from Saudi Arabia: a descriptive study, Lancet Infect. Dis. 13 (2013) 752-761.

[11] N. Lee, D. Hui, A. Wu, P. Chan, P. Cameron, G.M. Joynt, et al., A major outbreak of severe acute respiratory syndrome in Hong Kong, N. Engl. J. Med. 348 (2003) 19861994.

[12] K. Yuki, M. Fujiogi, S. Koutsogiannaki, COVID-19 pathophysiology: A review, Clin. Immuno., $215 \quad$ (2020) 108427, https://doi.org/10.1016/j.clim.2020.108427.

[13] P.A. Reyfman, J.M. Walter, N. Joshi, et al. Single-cell transcriptomic analysis of human lung provides insights into the pathobiology of pulmonary fibrosis. Am J Respir Crit Care Med (2019); 199: 1517-1536.

[14] R.J. Mason, Pathogenesis of COVID-19 from a cell biology perspective, Eur Respir J 2020; 55: 2000607, https://doi.org/10.1183/13993003.00607-2020.

[15] G. Li, X. Chen, A. Xu, Profile of specific antibodies to the SARS- associated coronavirus, N. Engl. J. Med. 349 (2003) 508509 , http://doi.org/10.1056/NEJM20030731349052 $\underline{0 .}$

[16] Mossel EC, Wang J, Jeffers S, et al. SARS-CoV replicates in primary human alveolar type II cell cultures but not in type Ilike cells. Virology 2008; 372: 127-135.

[17] Weinheimer VK, Becher A, Tonnies M, et al. Influenza $A$ viruses target type II pneumocytes in the human lung. J Infect Dis 2012; 206: 1685-1694. 
[18] Qian Z, Travanty EA, Oko L, et al. Innate immune response of human alveolar type II cells infected with severe acute respiratory syndrome-coronavirus. Am J Respir Cell Mol Biol 2013; 48: 742-748.
[19] C. Huang, Y. Wang, X. Li, et.al., Clinical features of patients infected with 2019 novel coronavirus in Wuhan, China, Lancet (2020), https://doi.org/10.1016/S0140-6736(20)30183$\underline{5}$. 